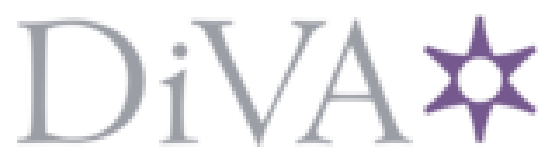

http://www.diva-portal.org

\title{
Postprint
}

This is the accepted version of a paper presented at IEEE PES GM 2015.

Citation for the original published paper:

Almas, M., Vanfretti, L. (2015)

RT-HIL Testing of an Excitation Control System for Oscillation Damping using External Stabilizing Signals.

In:

N.B. When citing this work, cite the original published paper.

Permanent link to this version:

http://urn.kb.se/resolve?urn=urn:nbn:se:kth:diva-170849 


\section{RT-HIL Testing of an Excitation Control System for Oscillation Damping using External Stabilizing Signals}

\author{
M. S. Almas \\ Electric Power Systems Department \\ KTH Royal Institute of Technology \\ Stockholm, Sweden \\ msalmas@kth.se
}

\author{
L. Vanfretti \\ KTH Royal Institute of Technology, Stockholm, Sweden \\ Statnett SF, Oslo, Norway \\ luigiv@kth.se, luigi.vanfretti@statnett.no
}

\begin{abstract}
A feature of an Excitation Control System (ECS) for synchronous generators is to enable power system stabilization by providing an additional input to the Automatic Voltage Regulator (AVR) for external stabilization signals. This paper explores this feature by externally generating stabilization signals which are fed as an analog input to a commercial ECS. This allows bypassing the built-in PSS function in the ECS and gives more freedom to the end-user to utilize custom stabilizer models. ABB's Unitrol 1020 Excitation Control System is coupled with Opal-RT's eMEGAsim Real-Time simulator to perform Hardware-in-the-Loop simulation of the ECS. The output of several stabilizer models is fed to the ABB's Unitrol 1020 ECS as external power system stabilization signals to analyze their performance for small signal stability enhancement.
\end{abstract}

Index Terms--Excitation Control System, Power System Stabilizer, Real-Time Simulation, Real-Time Hardware-in-theLoop Simulation, Opal-RT, Unitrol 1020, SmarTS-Lab, Power Oscillation Damping.

\section{INTRODUCTION}

An Excitation Control System (ECS) provides direct current to the synchronous machine field winding in order to regulate the generator's terminal voltage. In addition an ECS also provides protection functions to ensure that the capability limit of synchronous generators is never exceeded [1]. Some of the important features of an ECS are synchronous generator's terminal voltage control, over and under excitation limiters, field current limiters and protections [2].

One of the supplementary features of a commercial ECS is Power System Stabilization (PSS). This function provides adequate damping to the power system when small or large disturbances may result in undamped oscillations in an interconnected power system [3]. Undamped oscillations if not adequately addressed, result in loss of synchronism of one or group of machines from the rest of the power system and may cause the system to collapse [4]. Some commercial ECSs have the inherent functionality, where different vendors have implemented different PSS types in their ECS unit that can provide adequate damping for power system oscillations. However, this restricts the end-user to rely on the built-in PSS

This work was supported in part by Nordic Energy Research through the STRONg2rid project and by Statnett SF, the Norwegian TSO.

M. S. Almas, and L. Vanfretti are with KTH Royal Institute of Technology, Stockholm, Sweden. (e-mail: \{msalmas, luigiv $\} @ k t h . s e)$

L. Vanfretti, is with Statnett SF, Research and Development, Oslo, Norway (email: luigi.vanfretti@statnett.no) algorithms and does not give the freedom to use other more favorable damping input signals (if available) to the AVR of the ECS.

With the deployment of Phasor Measurement Units (PMUs) in the power grid, there is a possibility of utilizing wide-area measurements for damping power system oscillations [5]. Synchrophasor-based oscillation damping algorithms use either conventional PSS algorithms or phasorbased oscillation damping algorithms. In the first case, leadlag filters are used to provide phase compensation for the poorest damped mode. In the second case, phasor-based oscillation damping algorithms use the measured signal and separate it into its average and oscillatory component which is extracted in the form of phasor using either recursive techniques or low-pass filtering. The damping signal is computed by applying appropriate phase shift on the extracted oscillatory signal phasor [6].

The choice of oscillation damping algorithm deployed is dependent on several factors, e.g. available measurements, power system complexity, type of power damping device to be used, etc. [7]. These algorithms can be fed with either local measurements or wide-area measurements.

With the currently available commercial ECS, power system operators cannot select any wide-area measurements as an input damping signal to AVRs. The limitation is due to the fact that the built-in PSS implementation in the ECS only allows using local input measurements which are not user selectable. The only liberty provided to the user is to tune the parameters of the lead-lag filters to provide appropriate power oscillation damping, and this can only be done locally at the power plant and not remotely by the operator.

This paper explores the use of external damping signals in a commercial ECS to provide power oscillation damping, thus giving the freedom to utilize custom stabilizer models based on either local or wide-area measurements. A modified version of the 2-area 4-machine Klein-Roger-Kundur's [8] power system is executed in Real-Time using Opal-RT's eMEGAsim Real-Time Simulator (RTS) [9] and real-time hardware-in-the-loop simulation is performed using ABB's Unitrol 1020 Excitation Control System [10]. The external 
damping signals are provided by four different types of PSS models namely delta-speed $(\mathrm{d} \omega)$, dela-accelerating power $(\mathrm{dPa})$, multi-band pass PSS (MB-PSS) and a phasor based POD algorithm. The experimental performance assessment using RT-HIL simulation of this ECS for both terminal voltage regulation and power oscillation damping using builtin PSS was performed and presented in [11].

The paper is organized as follows: Section II provides information about the ABB's Excitation Control System Unitrol 1020 and test case modeling in MATLAB/Simulink. Section III presents the 4 different PSS models used to provide external damping signals to the ECS. RT-HIL simulation results for external damping signals as an input to ABB's ECS using Opal-RT's eMEGAsim real-time simulator for inter-area oscillation damping are discussed in Section-IV. In Section V, conclusions are drawn and future work is summarized.

\section{UNITROL 1020 OVERVIEW AND TEST CASE MODELING}

\section{A. Unitrol 1020 Excitation Control System}

Unitrol 1020 is an automatic voltage regulator (AVR) that provides excitation control of indirectly excited synchronous machines and rotors [10]. The primary purpose of the device is to maintain the generator's terminal voltage while taking into account all the operational limits associated to the generator. A typical generator receives mechanical power input from a turbine and its field excitation is provided by an excitation control system. The terminal voltage of the generator is fed to the excitation system which compares this value to the set-point (reference voltage) and computes required field current to bring the terminal voltage to the reference value.

\section{B. PSS Feature of Unitrol 1020 Excitation System}

The PSS feature available in Unitrol 1020 ECS is defined by the IEEE Std. 421.5-2005 PSS 2A/2B model [12] and its simplified representation is shown in Fig 1. The PSS2B has dual structures that use two signals of angular velocity " $\omega$ " and power "P" as compared to single input of angular velocity $\omega$ in PSS1A model. The RT-HIL based performance testing of this ECS for its built-in PSS is reported in [11]. In this study, external damping signals are provided from different PSS and POD algorithms to the AVR of the ECS to analyze the possibility of using custom power oscillation damping algorithms with a commercial ECS.

\section{Power System Modeling}

In order to investigate the performance of Unitrol 1020 when provided with external power system stabilization (PSS) signals, the Klein-Rogers-Kundur test system [8] was modeled in MATLAB/Simulink using the SimPowerSystems toolbox. The single line diagram of the test case is shown in Fig 2 displaying only Area-1 of the test case ${ }^{1}$. The test system consists of two fully symmetrical areas linked together by two $230 \mathrm{kV}$ lines of $220 \mathrm{~km}$ length.

\footnotetext{
${ }^{1}$ A complete single-line diagram of this test system can be found in [11]
}

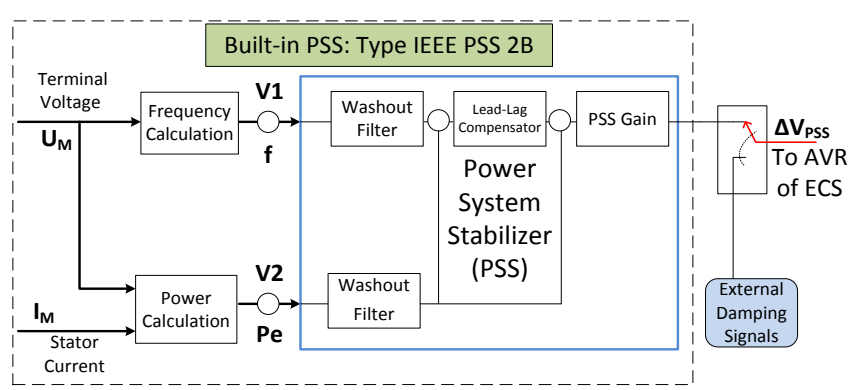

Fig. 1. Simplified model of the PSS implemented in Unitrol 1020 ECS. The calculated frequency and electric power Pe is fed to the PSS as voltage signals.

The excitation control of generator G1 is supplied through ABB's Unitrol 1020 ECS with its PSS feature enabled and configured to receive external damping signals. All other generators are equipped with IEEE Type-1 DC1A-DC commutator exciter model with no stabilization signal [12]. Therefore, damping for inter-area oscillations is provided by Unitrol 1020 ECS through external damping signals. In order to analyze the system response in presence of these external damping signals, a 5\% magnitude pulse was applied for 4 cycles at the voltage reference of generator G2 in Area 1 which results in an undamped oscillation of $0.64 \mathrm{~Hz}$ in the absence of any damping control.

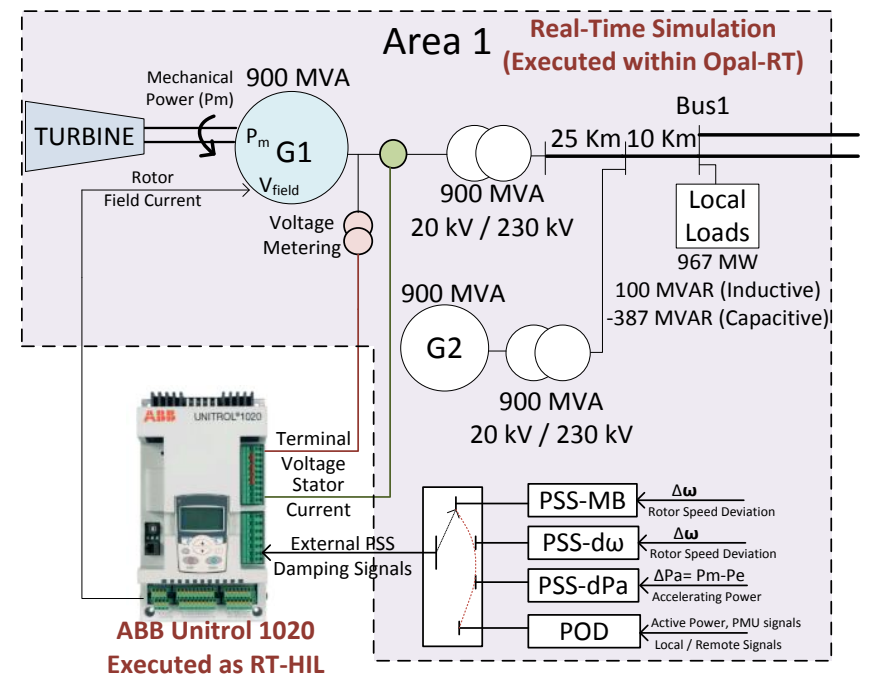

Fig. 2. Single line diagram of Klein-Rogers-Kundur's Power System showing only one Area. Only generator G1 is equipped with Unitrol 1020 ECS. All the different stabilizers models are executed in real-time in OpalRT. The only hardware interfaced with the simulator is Unitrol 1020 ECS.

\section{Interfacing Unitrol 1020 with Opal-RT's eMEGAsim Real-Time Simulator}

The Real-Time Simulator (RTS) used here can only provide voltages up to $\pm 10 \mathrm{~V}$ and currents up to $\pm 20 \mathrm{~mA}$. These low-level analog outputs (corresponding to generator terminal voltage and stator current) are amplified using linear amplifiers to scale voltage up to $100 \mathrm{~V}$ and currents to 1 Ampere at rated power [13]. The field current measurement is supplied to Unitrol 1020 using low-level \pm 10 Volts. For this purpose one of the inputs of Unitrol 1020 is configured for 
receiving external excitation current. Different PSS and POD models are executed in Opal-RT and their output damping signals are fed as an analog input to the ABB's Unitrol 1020 ECS. By utilizing CMT 1000 (Unitrol 1020 configuration software), the PSS feature was set to enable and the analog input being fed with external damping signals was configured as primary stabilizing signal to the ECS. The complete connection diagram is shown in Fig 3.

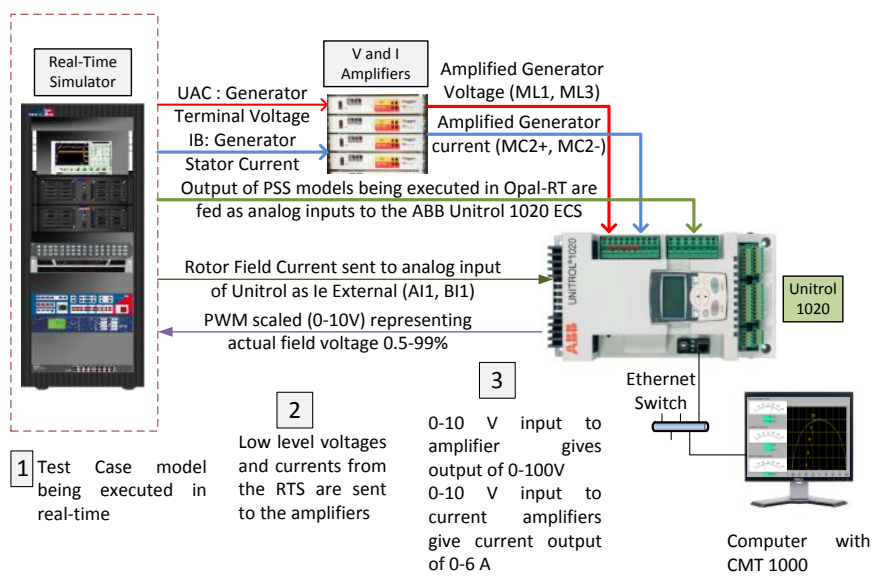

Fig. 3. Connection Diagram for interfacing Opal-RT with Unitrol 1020 ECS. The output of PSS/POD models being executed in real-time in Opal$\mathrm{RT}$ is fed to the ABB's Unitrol 1020 through its analog input configured for receiving external damping signals.

\section{RT-HIL ASSESSMENT WITH EXTERNAL DAMPING SIGNALS}

This section analyses the performance of the ECS for power system stabilization when provided with external damping signals from 4 different stabilizer models. A brief description of the different PSS models and their RT-HIL results are presented sequentially.

\section{A. Multi-Band PSS}

The IEEE type PSS4B PSS is described in the IEEE Standard 421.5 [12]. This block was implemented in the test case model and tuned to provide damping for the inter-area oscillation mode of $0.64 \mathrm{~Hz}$. The model is shown in Fig 4 and is presented in [14]. It consists of three bands namely low, intermediate and high band. The low band takes care of very slow oscillating phenomena (i.e. drift mode). The intermediate band is used for inter-area modes usually found in the range of 0.2 to $1.0 \mathrm{~Hz}$. The high band deals with local modes with a typical frequency range of 0.8 to $4.0 \mathrm{~Hz}$.

As shown in Fig 4, the input to the MB-PSS is the rotor speed deviation and output is a stabilizing signal which is provided as an external damping signal to the ABB's Unitrol 1020 ECS to provide adequate damping. The response of the test case model in presence of MB-PSS as an external damping signal for small disturbance (5\% step change in voltage reference of G2) is shown in Fig 5 in the magenta trace.

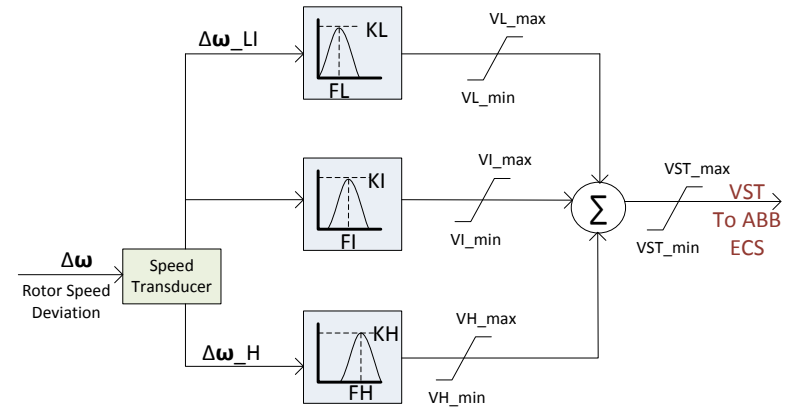

Fig. 4. MB-PSS simplified model with $\mathrm{K}_{\mathrm{L}}=30, \mathrm{~F}_{\mathrm{L}}=0.05 \mathrm{~Hz} ; \mathrm{K}_{\mathrm{i}}=40.0 \mathrm{~F}_{\mathrm{I}}=$ $0.80 \mathrm{~Hz} ; \mathrm{K}_{\mathrm{H}}=160.0 \mathrm{~F}_{\mathrm{H}}=8.0 \mathrm{~Hz}$

\section{B. Conventional Delta $\boldsymbol{\omega}$ PSS}

The simplest method to provide a damping torque in the synchronous machine is to measure the rotor speed and use it directly as an input signal in the stabilizer structure. This PSS is illustrated in Fig 6 and consists of a low-pass filter, a gain, a washout filter (which is effectively a high-pass filter), a phase-compensation (lead-lag filter), and an output limiter. The general gain " $\mathrm{K}$ " determines the amount of damping produced by the stabilizer. The washout high-pass filter allows the PSS to respond only to transient variations in the speed input signal "d $\boldsymbol{\omega}$ ". The response of the test case power system in presence of delta $\boldsymbol{\omega}$ PSS as external damping signal for small disturbance (5\% step change in voltage reference of G2) is shown in Fig 5 in red trace.

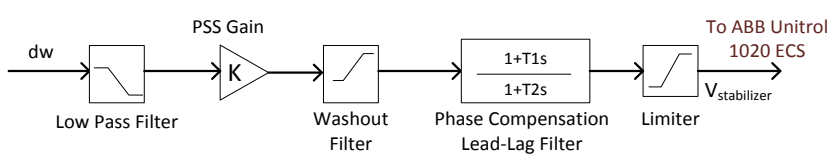

Fig. 6. Model of conventional $\Delta \boldsymbol{\omega}$ PSS [11]. PSS Gain (K) is set to 20, T1 and $\mathrm{T} 2$ for phase compensation are set to 0.05 and 0.02 respectively.

\section{Conventional Acceleration Power (Delta Pa) PSS}

The acceleration power of a generator is defined as the difference between its mechanical input power and electrical power output. This acceleration power is used as an input signal for the conventional PSS presented in Fig 6. The model of a Delta Pa PSS is shown in Fig 7. The response of the test case power system in presence of the delta Pa PSS as external damping signal for small disturbance $(5 \%$ step change in voltage reference of G2) is shown in Fig 5 in the green trace.

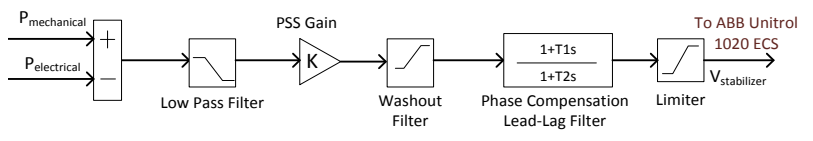

Fig. 7. Model of conventional $\triangle \mathrm{Pa}$ PSS. PSS Gain (K) is set to 3.5, T1 and $\mathrm{T} 2$ for phase compensation are set to 0.06 and 1 respectively.

\section{Power Oscillation Damper}

Another approach for generating damping signals is called phasor POD and proposed in [15]. The principle for creating 
the damping signal using the Phasor POD approach is shown in Fig 8. The main objective is to separate the oscillatory part of the input signal from the average value. The oscillatory part of the measured input signal is extracted as a phasor by using either a recursive least square estimation technique or a low pass filter. The damping signal is computed by applying an appropriate phase shift on the extracted oscillatory part of the signal.

The damping signal can be provided to the excitation control system of synchronous generators to provide oscillation damping. The flexibility of this algorithm with respect to input signals make it simple to use any synchrophasor based wide-area measurement as input. For this study, the active power flow at the midpoint of the test case system is used as an input to phasor POD algorithm, however, wide-area measurements like voltage magnitude/phase angle and current magnitude/phase angle can be used similarly by applying appropriate phase shift. The response of the test case power system in presence of Phasor POD as an external damping signal to Unitrol 1020 ECS for small disturbance $(5 \%$ step change in voltage reference of G2) is shown in Fig 5 in the blue trace.

\section{DISCUSSION ON SIMULATION RESULTS}

In the absence of any external PSS or POD signal to Unitrol 1020, the system is unstable (cyan trace in Fig 5) and the $0.64 \mathrm{~Hz}$ oscillatory mode is visible in the active power

\section{Rotor Field Current Input to Unitrol 1020 ECS}
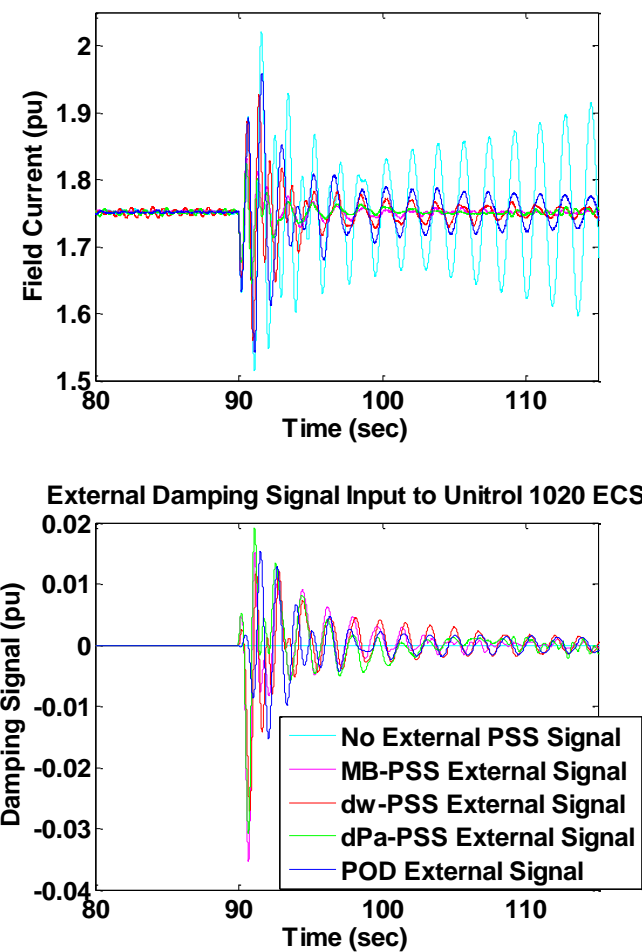

transfer between Area 1 and Area 2 when the small disturbance is applied. All the external damping signals provide adequate damping for $0.64 \mathrm{~Hz}$ mode. The external

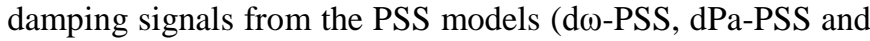
MB-PSS) provide much better power oscillation damping as compared to the phasor based power oscillation damping signal because their lead-lag filters were effectively tuned to provide phase compensation for the $0.64 \mathrm{~Hz}$ mode.

The phasor POD based external damping signal results in complete oscillation damping in $40 \mathrm{sec}$. The slower response of phasor POD (blue trace) in damping out the $0.64 \mathrm{~Hz}$ mode

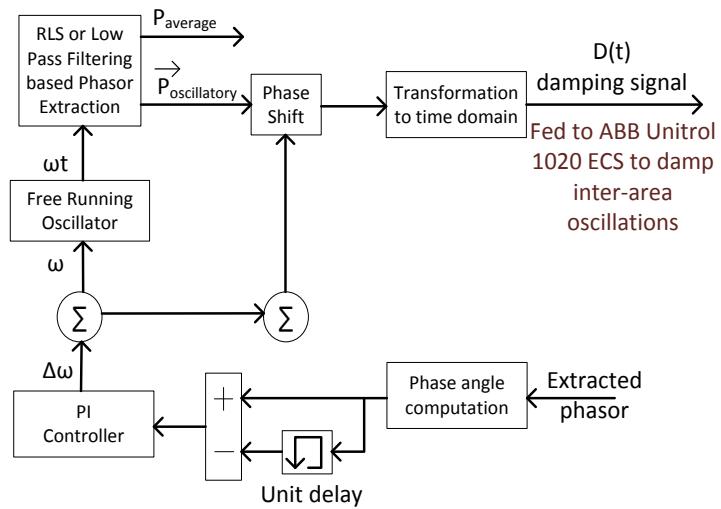

Fig. 8. Operating principle of Phasor POD and generation of damping signal which is fed to Unitrol 1020 ECS as external damping signals.

Field Voltage Output from Unitrol 1020 ECS to Generato
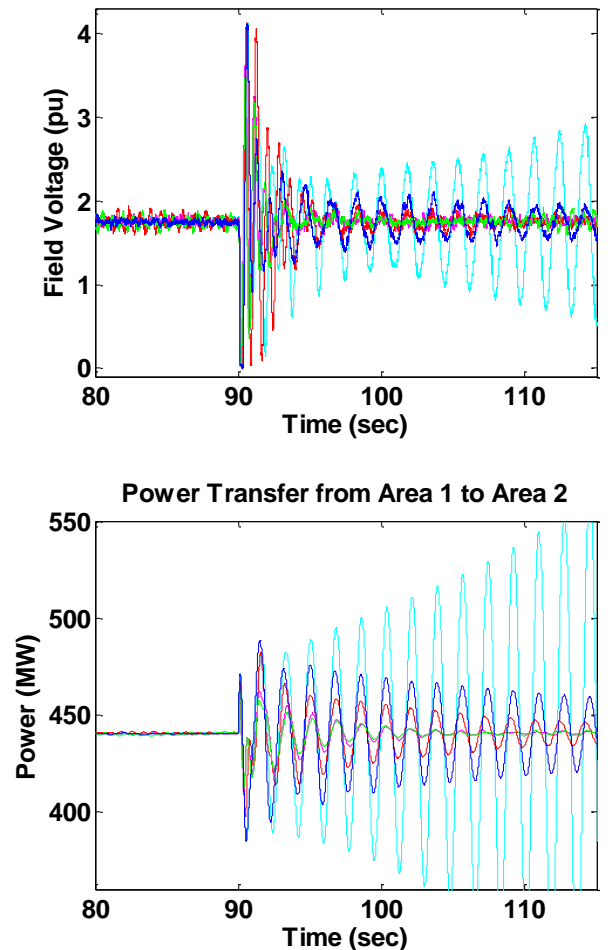

Fig. 5. Response of Test Case model when a $5 \%$ magnitude step is applied at the reference voltage of Generator 2 at t=90 sec for 10 cycles. Only generator's G1 parameters are shown in this figure when Unitrol 1020 ECS is fed with different external damping signals. (Top-Left) shows rotor field current of Generator G1 which is fed to Unitrol 1020 ECS, (Top-Right) shows the field voltage output from Unitrol 1020 ECS which is fed back to the generator G1 model being executed in real-time in Opal-RT, (Bottom-Left) shows the output of different PSS/POD models when the disturbance is introduced in test case system. These external damping signals are fed to Unitrol 1020 ECS for power system stabilization. (Bottom-Right) shows power transfer from Area 1 to Area 2 where the inter-area oscillation of $0.64 \mathrm{~Hz}$ is adequately damped by Unitrol $1020 \mathrm{ECS}$ using external damping signals. 
relatively low, so that output damping signal does not violate the operational limits of the Unitrol 1020 ECS and the generator G1 to which it is coupled. This contradicts the results in [6], which indicate an error in the experimental test. This experiment should be repeated by introducing saturation limits to the output of the POD and increasing the gain.

The field voltage analog input signal from Unitrol 1020 has noise. This can be countered in the simulation by adding a discrete mean block which computes the average of this analog input and then feeds it to the input of the generator model. Some of the numerical noise is product of the scaling of the signals, which is needed to remain within the threshold limits of Opal-RT's analog outputs, amplifiers inputs and analog inputs of the Unitrol 1020 ECS. These scalings cannot be avoided and results in the small variation in the field voltage output by Unitrol 1020 even in steady-state (Fig 5Top Right). Figure 9 shows the screenshot of the oscillography obtained from CMT 1000 (Unitrol 1020 Configuration Software) while performing the test with phasor POD based damping signal as an external stabilizing signal to the PSS.

\section{CONCLUSION AND FUTURE WORK}

The stabilization capability of a commercial ECS is analyzed by providing it with external damping signals from different stabilizer models. RT-HIL simulation is performed for a 2-area 4-machine test system using Opal-RT real-time simulator and one of the generator in the test system is equipped with ABB's Unitrol 1020 ECS. The RT-HIL results have shown that the external damping signals from different stabilizer models can be fed to the commercial ECS to provide adequate damping to inter-area oscillations and thus liberating the users from the restriction of relying only on the built-in PSS implementation of the ECS.

Further work will be focused on using different types of real PMU signals from an RT-HIL setup as input to an external hardware-based stabilizer implementation as experimental proof of the theoretical results in [16]. These results will be submitted in a future publication.

\section{ACKNOWLEDGMENT}

This work was financially supported in part by Nordic Energy Research through the STRONg ${ }^{2}$ rid project and by Statnett SF, the Norwegian Power System Operator. KTH SmarTS-Lab would like to thank ABB Switzerland AG for their donation of Unitrol 1020 Excitation System.

\section{REFERENCES}

[1] F.P. deMello and C. Concordia "Concepts of synchronous machine stability as affected by excitation control", IEEE Trans. on Power Apparatus and Systems, vol. 88, no. 4, pp.316 -329, 1969

[2] IEEE Guide for "Synchronous Generator Modelling Practices in Stability Analyses,", Power System Engineering and Electric Machinery Committees of the IEEE Power Engineering Society, IEEE Std.1110-1991

[3] F.P. deMello and T.F. Laskowski "Concepts of power system dynamic stability", IEEE Trans. on Power Apparatus and Systems, vol. 94 , pp.827 -833,1979

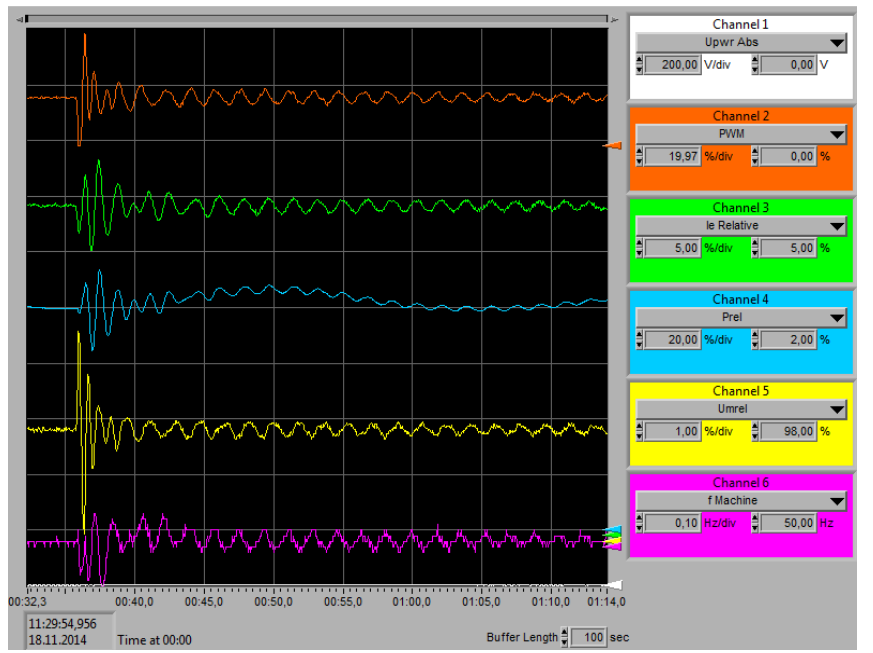

Fig. 9. The figure shows field voltage output from Unitrol 1020 ECS (red trace), stator current of G1 (green trace), active power as computed at the terminal of the generator G1 by ECS (blue trace), terminal voltage of G1 (yellow trace) and frequency of G1 (magenta trace).

[4] M. Klein , G. Rogers and P. Kundur "A fundamental study of interarea oscillations in power systems", IEEE Trans. On Power Systems, vol. 6, no. 3, pp.914-921 1991

[5] K. Uhlen, L. Vanfretti, M.M. de Oliveira, A. B. Leirbukt, V. H. Aarstrand, and J.O. Gjerde, "Wide-Area Power Oscillation Damper Implementation and Testing in the Norwegian Transmission Network," IEEE PES General Meeting 2012, San Diego, CA, USA.

[6] M. S. Almas and L. Vanfretti, "Implementation of Conventional and Phasor Based Power System Stabilizing Controls for Real-Time Simulation", 40th Annual Conference of IEEE Industrial Electronics Society (IECON), Dallas, USA, Oct 29-Nov 01, 2014

[7] N. R. Chaudhuri, S. Ray, R. Majumder and B. Chaudhuri "A case study on challenges for robust wide-area phasor POD", Proc. IEEE Power Eng. Soc. General Meeting, 2009

[8] P. Kundur; M. Klein G. J. Rogers and M. S. Zywno, "Application of Power System Stabilizers forEnhancement of Overall System Stability", IEEE Transactions on Power Systems, Vol. 4, No. 2, August 1989, pp. 614-626

[9] Opal-RT, "eMEGAsim PowerGrid Real-Time Digital Hardware in the Loop Simulator", Available online: http://www.opal-rt.com/.

[10] ABB-Unitrol 1020 Automatic Voltage Regulator, available online: http://tinyurl.com/Unitrol

[11] M. S. Almas and L. Vanfretti, "Experimental Performance Assessment of a Generator's Excitation Control System using Real-Time Hardware-in-the-Loop Simulation", 40th Annual Conference of IEEE Industrial Electronics Society (IECON), Dallas, USA, Oct 29-Nov 01, 2014.

[12] IEEE Standard 421.5-2005, "IEEE Recommended Practice for Excitation System Models for Power System Stability Studies", 2006, DOI: 10.1109/IEEESTD.2006.99499

[13] "Current and Voltage Amplifiers by Megger," available on-line: http://www.megger.com/cae/story/Index.php?ID=527.

[14] R: Grondin, I. Kamwa, et.al, “ Modeling and closed-loop validation of a new PSS concept, the multi-band PSS", IEEE PES General Meeting 2003, Toronto, Canada

[15] L. Angquist, B. Lundin and J. Samuelsson, "Power oscillation damping using controlled reactive power compensation-a comparison between series and shunt approaches", IEEE Transactions on Power Systems, Vol. 8 , No. 2, July 1993 , pp. $687-700$

[16] Y. Chompoobutrgool and L. Vanfretti, "Identification of Power System Dominant Inter-Area Oscillation Paths," IEEE Transactions on Power Systems, vol. 28, no. 3, pp. 2798-2807, Aug. 2013 